\title{
A New Algorithm Design for the Real-time Electrochemical Impedance Monitoring System
}

\author{
Byoung-Yong Chang ${ }^{\dagger}$ \\ Department of Chemistry, Pukyong National University, 45 Yongso-ro, Nam-gu, Busan 608-739, Korea
}

\begin{abstract}
:
It is generally known that electrochemical impedance spectroscopy is a powerful technique and its real-time application has been demanded for prompt observations on instantaneous electrochemical changes. Nevertheless, long measurement time and laborious analysis procedures have hindered development of it. Solving the problems, here I report of a new algorithm design for development of a real-time electrochemical impedance monitoring system, which potentially provides a guideline in developing monitoring systems of electric vehicles batteries and other electrochemical power plants. The significant progress in this report is employment of the parallel processing protocol which connects independent sub functions to successfully operate with avoiding mutual interruptions. Therefore, all the processes required to monitor electrochemical impedance changes in realtime are properly operated. To realize the conceptual scheme, a Labview program was coded with sub functions units which conduct their processes individually and only data are transferred between them through the parallel pipelines. Finally, measured impedance spectra and analysis results are displayed, which are synchronized according to the time of change.
\end{abstract}

Keywords: Impedance spectroscopy, Electrochemical instrument, Real-time measurement

Received November 6, 2012 : Accepted December 29, 2012

\section{Introduction}

Electrochemical techniques provide handy platforms for measuring chemical changes because they directly convert chemical changes to electric signals. ${ }^{1)}$ Normally, chemical measurement devices employ additional transducers to convert chemical energy perturbed by chemical processes to electric analog/digital signals which instruments can handle with. For example, absorption spectrum related to chemical bonds and reactions are obtained by a photon detector. ${ }^{2}$ Here, the photon detector, as the transducer, converts the chemical information to electronic signals by counting the photons. However, in electrochemistry, because chemical reactions are interfaced with elec-

${ }^{\dagger}$ Corresponding author. Tel.: +82-51-629-5597

E-mail address: bychang@pknu.ac.kr trode, chemical information is directly transferred to the electrode which produces electric signals and transmits to the display instrument. ${ }^{3)}$ Therefore, we can remove complexity of chemical instrumentation and processes as well as enhance performance and portability.

There are many electrochemical techniques to observe chemical phenomena. Constant or scanning potential methods are very frequently used. They can report much electrochemical information even with a simple relationship between potential and current. Yet, the more complex the chemical phenomena are, the higher resoluble methods are needed. One of the advanced techniques is electrochemical impedance spectroscopy (EIS), which is very sensitive to chemistry occurring at the electrified interface. ${ }^{4)}$

The fundamental of EIS resides in use of ac signals of various frequencies. The electrochemical processes 
at the electrode/electrolyte interface are complexly composed, so that a simple $E-I$ curve cannot provide clear vision of such complexity. ${ }^{3)}$ However, taking advantage of that those processes respond differently according to frequencies, we could find ways to resolve the complex relationships among the processes. For instance, if we are interested in the electrochemical behavior of a specific process such as electron transfer, mass transfer, and electric double layer formation, we can aim at studying the responses upon ac waves of an appropriate frequency, on which other processes do not depend. ${ }^{5)}$ Another example is study on the collective frequency behavior made by the target chemical reaction. It is already known that diffusion of molecules can be studied by plotting the imaginary number of impedance against $1 /$ (frequency ${ }^{1 / 2}{ }^{13)}$ Also, the phase angle changed along frequencies tells capacitance and response times at differently treated electrodes. ${ }^{6,7)}$ Still, EIS does more things; it can describe the complex electrochemical phenomena as an equivalent circuit. The equivalent circuit consists of all the possible processes involved, so that the whole picture of electrochemistry can be drawn. ${ }^{8}$ In other words, the obtainable information is not limited to specific ones, but extends to the complex relationships among multiple faradaic and nonfaradaic processes.

That method can be achieved by means of the nonlinear-curve-fitting (NLCF) technique. When possible equivalent circuits are proposed, the NLCF fits the measured EIS spectrum to the circuit to evaluate the components in the circuit. Here, the components are analogous to electrochemical processes, thus we are able to find the values of thermodynamic and kinetic parameters based on the equivalent circuit. ${ }^{4,9)}$ The NLCF is a widely used method to result in the best values fitting to given conditions, and available commercially. However, its algorithm costs much mathematical resources due to rigorous calculations, so that a practical application of NLCF to impedance analysis is restricted. Therefore, impedance measurement and analysis should be made separately, and real-time impedance monitoring is hardly accomplished.

Another hurdle for real-time impedance monitoring system is that impedance spectrum cannot be obtained in the transient state. ${ }^{10-12)}$ In an experiment, when a bias potential at which electrochemical reactions take place is applied, a certain amount of time should be waited until the steady state is reached. Then, ac wave perturbations are applied to drive ac responses of electrochemical processes. Here, the desired bias potential application and ac perturbation are not synchronized. Consequently, real-time impedance acquisition is inherently impossible. However, a recent progress of EIS solved this problem. Chang and Park developed theories about transformation of dc to ac electrochemical signals leading to electrochemical impedance as function of frequency. ${ }^{13)}$ According to their method, ac perturbation is not needed, and only a bias potential application is used to measure impedance spectrum of a wide frequency range. Thus, the measured impedance can be synchronized to the applied potential, which promises the real-time impedance monitoring system.

The real-time impedance monitoring system has been continuously demanded because simple $E-I$ relationships have limitations to fully monitor on-going electrochemical processes. Nonetheless, the real-time impedance monitoring system has not been accomplished due to the obstacles explained above. ${ }^{8,14)}$

Here in this report, I will describe how I designed a program to conduct the real-time impedance monitoring by improving the previous FTEIS system to carry out rigorous measurement and analysis simultaneously.

\section{Experimental}

A potentiostat capable of handling high speed signals with high precision is home-made and connected to a NI PCI-4461 card (National Instruments, Austin, TX) installed in a PC. The card is called a dynamic signal analyzer (DSA), and functions as an arbitrary waveform generator through its output terminals, and a signal digitizer acquiring data through the input terminals. The DSA board generates a staircase waveform as the source of high speed potential steps transmitted to the potentiostat. The potentiostat stably delivers them to the experiment cell. (In this report, only a dummy cell consisting of a capacitor $(10 \mu \mathrm{F})$ and two resistors $(1 \mathrm{k} \Omega)$ is used for demonstration instead of an electrochemical cell.) The current and responding potential data are acquired at $204.8 \mathrm{k}$ samples using the DSA board. Impedances calculation and non-linear curve fitting simulation are carried out in a single Labview program composed of many sub function units. To the purpose of verifying my new proposal, two Labview programs are coded with and 
without the parallel processing protocol. Here, the core sub function units are the analog waveform generator, the signal digitizer, the FFT impedance calculator, and the analyzer in Fig. 1, and are connected via serial and parallel pipeline as shown in the figures. The former two function units are provided by NI and the latter two are home-made. The FFT impedance calculator transforms the dc current and potential to ac impedance based on the algorithm published before. Briefly, the transient currents and potentials recorded in the time domain are differentiated $[I(t), E(t) \rightarrow \Delta I(t)$ and $\Delta E(t)]$, and Fourier transformation transfers the data to the frequency domain $[\Delta I(t)$ and $\Delta E(t) \rightarrow$ $\Delta I(\omega)$ and $\Delta E(\omega)]$, Finally, Impedances, $Z(\omega)$, are calculated by $\Delta V(\omega) / \Delta I(\omega)$. More details are described elsewhere. ${ }^{8)}$

\section{Results and Discussion}

The existing FTEIS measurement protocols are following; (1) step potential waveforms are applied to the electrochemical cell, (2) the applied potential waves and the resulting currents are acquired by a digitizer installed in a PC and recorded on a hard disk drive, (3) the recorded potentials and currents undergo the mathematical operation; differentiation, Fourier transform, and impedance calculation, and (4) analysis based on the non-linear-curve-fitting to equivalent circuits is performed to extract electrochemical information from impedance spectrum. Step (1) and (2) refer to realtime recording of potential and current done by the waveform generator and the digitizer in the PC, but Step (3) and (4) belong to the calculation part that exhaust the resources of the PC such as CPU performance and internal/external data transfer. Even though Step (1) and (2) can be done simultaneously, Step (3) and (4) should be done separately one by one. In the pursuit of the real-time impedance monitoring system, Step (1) through (4) should be done without time lag not to mention accuracy of measurement. Unfortunately, even the state-of-art computing system cannot handle the whole (1) to (4) sequential processes.

However, owing to the computing system advancing to the multi-tasking platforms with multi-core CPUs, we find a roundabout way to handle the whole processes without time lag. The key point of this work is the parallel computing operation. Step (1) and (2) are done regularly, and the data are transferred to another memory area to be ready to be subject to Step
(3). Once the data are transferred, Step (1) and (2) are safe to conduct the next data acquisition without time lag. Because the rate of data transfer is much faster than that of data acquisition, the data is neither delayed nor missing while Step (1) and (2) carry out continuously. Step (3) carries out the rigorous calculation to result in impedance spectrum from the transferred data. Especially, Fourier transform requires a long time with full load on the CPU, which affects the overall performance of FTEIS measurement. However, as the present computing system supports the multi-core processors for multi-tasking, we can separate the roles of data acquisition and calculation to be carried out simultaneously without interference on the overall performance. This multi-tasking can be applied to Step (4) as well. In summary, we can realize the real-time impedance monitoring system based on the parallel operations of data acquisition, calculation, and analysis, which are connected only by data transfer not by processing sequence.

In order to fulfill the parallel operation, we approached from the viewpoint of software rather than hardware. Because Labview from National Instrument (NI) provides such parallel computing platforms optimized to multi-core CPUs as well as high-speed data acquisition, we managed to code a Labview program to synchronize and carry out the (1) to (4) processes. Fig. 1(a) shows the graphical program that contains sub function units performing Step (1) to (4) in the sequential manner as explained above. Fig. 1(b) shows a modified program that carries out the same functions but the sub units work in the parallel manner. Here, the sub units communicate each other with only data transfer. The program without the parallel processing handles the data acquisition and the calculation one by one, so that each process cannot be executed until the others are finished. For example, when the first data acquisition is finished, the second data acquisition cannot be executed without completing the calculation, which will cause the time delay between each data acquisition process. In that situation, accurate impedance monitoring is not guaranteed. On the other hand, the program with the parallel processing handles each process independently. Once the data acquired by block (2), block A saves and hands over the data to block (3) while the next data are acquired by block (2) whether or not the first data are calculated by block (3). Block (2) continuously acquires the data by itself regardless the other processes. Block (3) only func- 

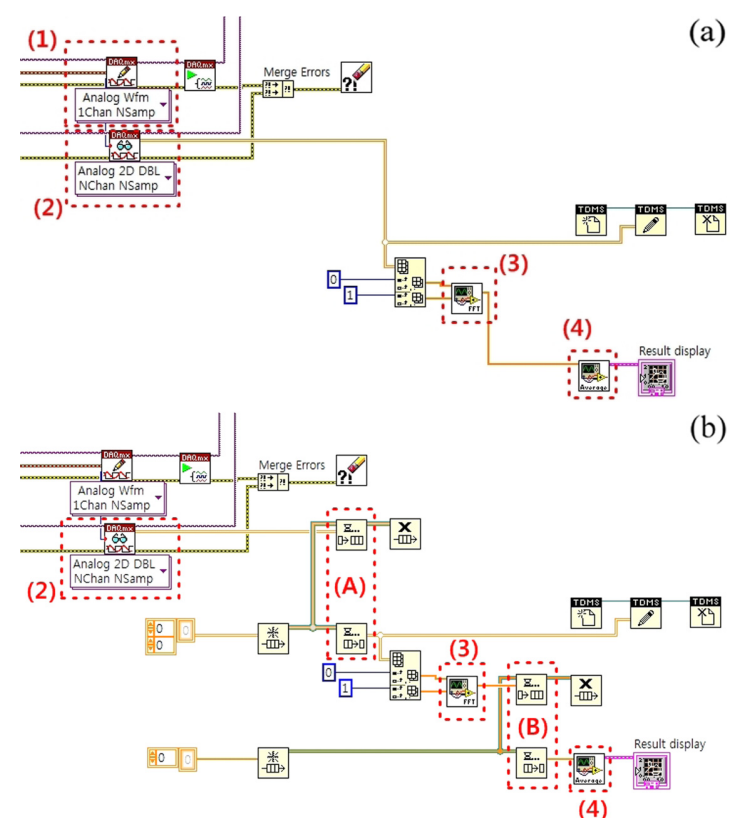

Fig. 1. Labview graphical programs which carry out the whole impedance spectroscopy procedure from measurement to analysis are shown (a) without and (b) with the parallel processing designs. Step(1): staircase waveform application, step(2): current and potential signal acquisition, step(3): impedance calculation through Fourier transform, and step(4): analysis of the impedance results. Block (A) and (B) have parallel pipeline for data transfer between above steps.

tions the impedance calculation, then the impedance results are transmitted by block (B) to the NLCF module (block 4) while data are acquired by block (2) and calculated by block (3), respectively. When the NLCF module receives the impedance spectrum, it starts to fit the data to the appropriate equivalent circuit to evaluate the electrochemical parameters. Eventually, the measured and calculated data are displayed. As the rate of data transfer is much faster than any of the processes, there is no interference possibly made during the data transfer.

Fig. 2 shows some of sequential currents obtained upon sequential potential steps without and with the parallel algorithm. Even though we apply step potentials and sampled the resulting signals with the same rates and intervals, we do not manage to acquire the whole complete sets of synchronized currents and potentials. That means that there must be some delayed/missing data when acquired. Since Fourier transform is very dependent on the time coordinate, even a delayed point can distort the whole impedance
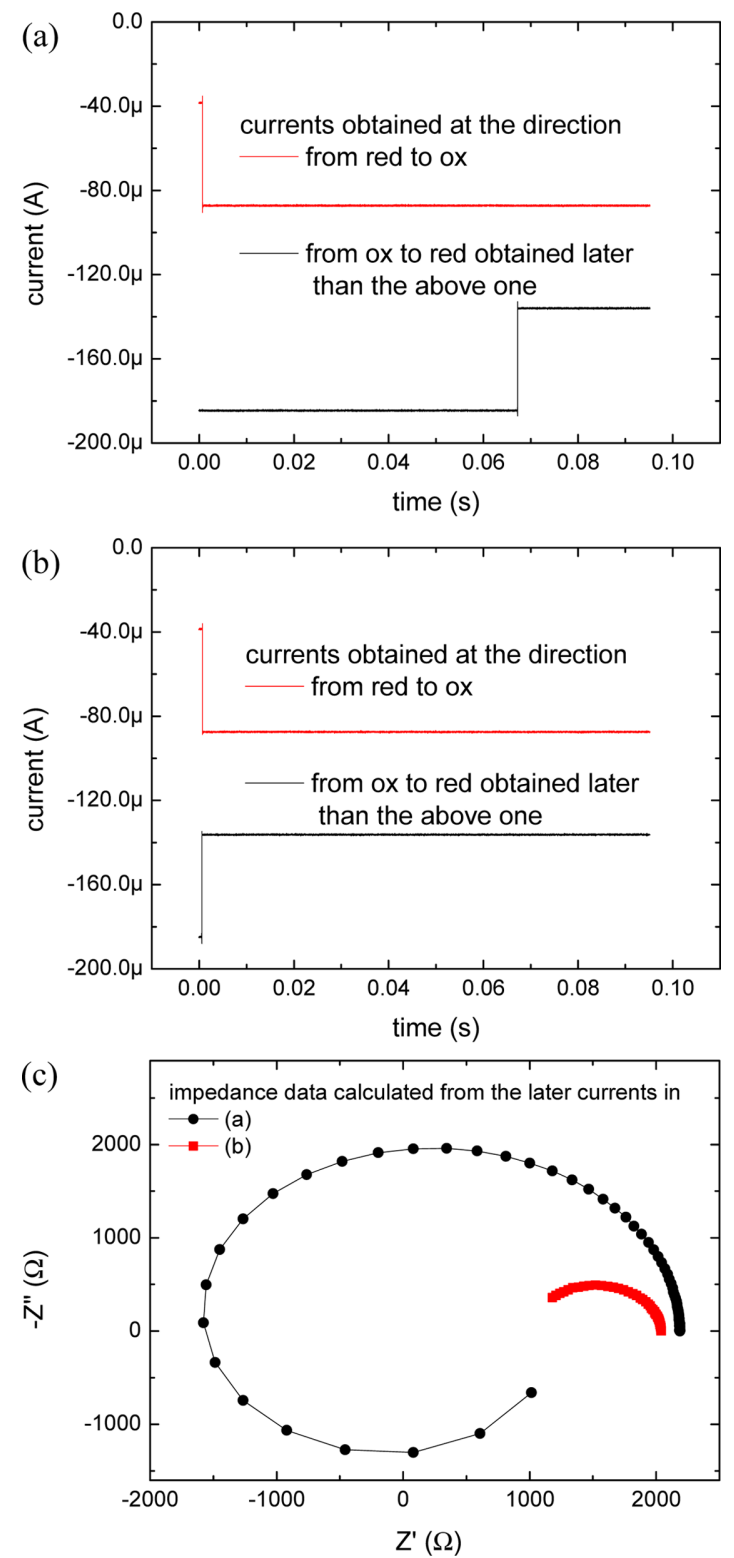

Fig. 2. Experimental results are obtained with a dummy cell using the above programs (a) without and (b) with the parallel processing designs. The top lines were obtained at the beginning of the FTEIS experiment and the lower lines were obtained one second later than the beginning. (c) Nyquist plots of impedance spectrum calculated from the later currents, black lines in (a) and (b), are compared.

spectrum. However, when the processes are carried out in parallel, the complete sets of data were obtained without a single missing point, and all data are accurately synchronized. 


\section{Conclusion}

Among the electrochemical measurement techniques, electrochemical impedance spectroscopy is the most sensitive one to resolve even complex reactions involving multiple processes. The cost of such advantage is long measurement time and laborious work on data analysis. The time problem had been solved by the Fourier transform method; the currents upon the sequential dc potentials are directly converted to ac impedance spectrum, so that the applied potential and the obtained impedance are synchronized. While the impedance spectra are acquired in real-time by the Fourier transform method, calculation and analysis of the data for real-time monitoring was not possible due to the high resource consumption which interferes continuous data acquisition. In this report, we suggest a new algorithm to solve that problem. Instead of serial processing of the data acquisitions and calculations, parallel processing of those steps are employed in the operating program. Once the data are transferred to one processing unit, the data are tossed again to the next unit before the time is spent by that processing unit. Then, processing units do not interfere with each other, impedance spectra and their final results are possible to monitor. This new design is expected to provide development guides for monitoring systems of electric vehicle batteries, ${ }^{15)}$ fuel cell power plants, ${ }^{16,17)}$ and so on.

\section{Acknowledgment}

This research was supported by Basic Science Research Program through the National Research Foundation of Korea (NRF) funded by the Ministry of Education, Science and Technology (2011-0009714).

\section{References}

1. B.-Y. Chang and S.-M. Park, Annu. Rev. Anal. Chem. 3, 207-229 (2010).

2. D. A. Skoog, F. J. Holler and T. A. Nieman, Principles of Instrumental Analysis. 5ed. Harcour Brace \& Company, Orlando (1998).

3. A. J. Bard and L. R. Faulkner, Electrochemical Methods: Fundamentals and Applications. Wiley, New York (2002).

4. E. Barsoukov and J. R. Macdonald, Impedance spectroscopy: theory, experiment, and applications. $2 \mathrm{ed}$. Wiley-Interscience, (2005).

5. M. Choi, K. Jo and H. Yang, J. Electrochem. Sci. Tech. 3, 24-28 (2012).

6. J. H. Nam, C. H. Woo, K. M. Kim, K. S. Ryu and J. M. Ko, J. Electrochem. Sci. Tech. 3, 80-84 (2012).

7. D. E. Smith, Anal. Chem. 35, 610-614 (1963).

8. B. Y. Chang, E. Ahn and S. M. Park, J. Phys. Chem. C 112, 16902-16909 (2008).

9. A. Lasia, Electrochemical Impedance Spectroscopy and its applications. In Modern Aspects of Electrochemistry, White, R. E.; Conway, B. E.; Bockris, J. O. M., Eds. Plenum Press: New York, Vol. 32 (1999).

10. M. Sluyters-Rehbach and J. H. Sluyters, J. Electroanal. Chem. 102, 415-419 (1979).

11. Z. B. Stoynov, Electrochim. Acta 37, 2357-2359 (1992).

12. G. S. Popkirov and R. N. Schindler, Electrochim. Acta 38, 861-867 (1993).

13. B.-Y. Chang and S.-M. Park, Anal. Chem. 79, $4892-4899$ (2007).

14. B.-Y. Chang, H. J. Lee and S.-M. Park, Electroanalysis 23, 2070-2078 (2011).

15. S. Rodrigues, N. Munichandraiah and A. K. Shukla, J. Power Sources 87, 12-20 (2000).

16. S. Andreasen, J. Jespersen, E. Schaltz and S. Kær, Fuel Cells 9, 463-473 (2009).

17. K. C. Hess, W. K. Epting and S. Litster, Anal. Chem. 83, 9492-9498 (2011). 